\title{
A Distributed nodes' localisation approach in Wireless Sensor Networks
}

\author{
Y. Hamam, Senior Member, IEEE, K. Djouani, Member, IEEE, and O.J. Oyedapo \\ F'SATI at TUT, Pretoria, South Africa \\ Tshwane University of Technology \\ Private Bag X680 \\ Pretoria 0001, Republic of South Africa \\ Email: \{hamama,djouanik,oyedapoo\}@tut.ac.za
}

\begin{abstract}
Node location is of main importance for several areas of applications based on Wireless Sensor Networks (WSNs), for instance location-based services. In this paper, we propose a new distributed approach for nodes'position estimation based on distance measurements. Our approach is close to the cooperative approach based on Curvilinear Component Analysis (CCA) proposed in [13].

The complexity of the proposed approach is $O(N)$ for all the network, but as the approach is distributed, the computation time is quasi-constant per node in the network.
\end{abstract}

\section{INTRODUCTION}

Localisation is the process of estimating the location or position of a wireless sensor node (WSN) within a sensor field. In some WSN applications, it may be required that sensor nodes learn their locations especially when the sensed data are required to be associated with this location information, known as location based services. For such application, localisation may be used to identify location of origin (position targeting during tracking) of sensed information as in the location of earthquake survivors and casualties. Localisation find its application in the search and rescue, disaster relief, target tracking, smart environment etc. Besides, geographical routing is based on location and nodes connectivity graphs. An efficient nodes' location technique should improve existing routing techniques in WSNs, by giving lower energy bound as a metric for routes discovery and network load balancing.

Localisation algorithms are broadly divided into two broad types-centralised and distributed algorithm. Centralised algorithm depend on a single sensor node equipped with a relatively greater computational resources. The data on the whole sensor network are gathered on this central node which, now compute and estimate the position of every node in the network and then ultimately disseminate its estimated location information back to the network. In distributed algorithm, individual nodes exchange signaling messages such that location estimations that were calculated in autonomous manner and propagated to their neighbours. Centralized and distributed localisation algorithm can be compared from the following perspectives [1], [12], [15], [13]:

(1) location estimation accuracy,
(2) implementation,

(3) computation issues and

(4) energy consumption.

It is noted that distributed localisation algorithms are strictly harder than the centralised in a sense that the distributed localisation can be applied to the centralised localisation problem but not the reverse [13].

The taxonomy for the localization of sensor node is thus highlighted. The distributed localization algorithms can be sub-divided into range-based, RSS based, and range-free techniques. The range-based consist of time of arrival (TOA), time difference of arrival (TDOA), and angle of arrival (AOA) techniques. It has been proved that the range-based technique can reach fine resolution but they require expensive hardware such as ultrasound device for the TDOA an antenna array for the AOA. Furthermore, the results of this technique depends on other unrealistic assumptions about signal propagation. If the hardware limitations of the sensor nodes which, translates to higher cost of the nodes is the main consideration for a specific WSN, then the range-free techniques are a cost effective alternative to the more expensive range-based techniques.

\section{RELATED WORKS}

Several approaches have been proposed recently to solve the problem of sensor localisation in adhoc wireless networks. An extension review of the proposed approach in the literature has been given in [1]. Triangulation and optimisation based methods are among the main methods. The earlier user neighbouring distance measurement for local estimation of the node's position and iterative steps to localise all nodes. For optimisation based approaches, the earlier work was reported by Doherty et al in [2].

Cost function is generally related to fitting the neighbouring nodes' Euclidean distance to the distance measurements :

$$
\left\|x_{i}-x_{j}\right\| \approx d_{i j}
$$

In [1], the authors propose SpaseLoc, a rule based algorithm for nodes'localisation in large scale WSNs. SpaseLoc proceed by solving the overall problem as a sequence of small convex optimization problem using Semi-definite Programming (SDP), in order to overcome the scalability issue with SDP. This approach is, in some sens, an extension of previous 


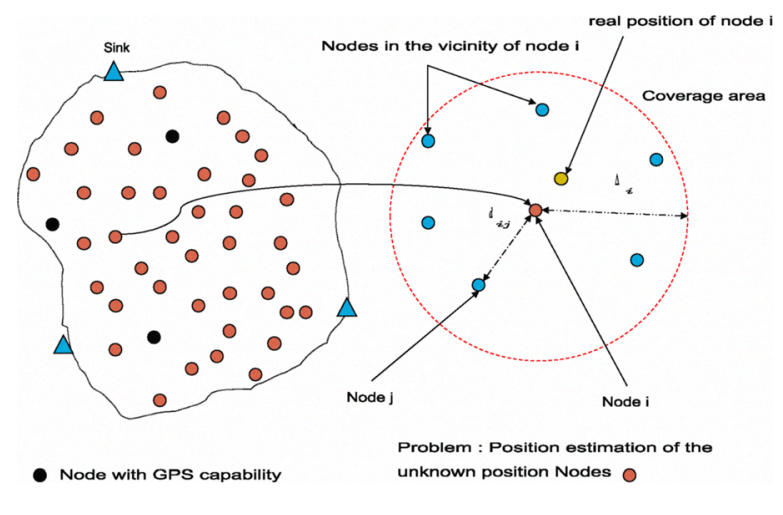

Fig. 1. WSN architecture and nodes' localisation Problem

published work on optimization based techniques [2], [3], [4] and [5]. The authors report a quasi linear complexity, but no discussion was reported on the clustering phase and the adaptive approach they propose for this case.

Elsewhere, the motivation for range-free localization algorithm is substantiated by its cost-effectiveness and reduced form factor. We are interested in range-free localization algorithms and RSS based algorithms that have been developed for WSN with strong emphasis on distributed nature of these algorithms. The DV-Hop algorithm [11], [7] is a hop-countbased localization technique where limited fraction of nodes have self positioning capability but the localization accuracy is poor. The Monte Carlo Localization (MCL) scheme, [8], is designed to obtain the probabilistic distribution of the node's possible location by using sequential Monte Carlo (SMC) that provide a simulation-based solution used to estimate the posterior distribution of non-linear discrete time dynamic models. Effectively, it represents posterior distribution of a sample and performs the update of these samples (once new observations are received) recursively in time using the importance method sampling. Initialization, prediction, filtering and update phases describe the MCL scheme. The MCL described in [8] makes use of the sequential Monte Carlo methods and uses mobility of nodes to attain good accuracy but suffers large latency because of the computation-intensive sampling methods used and the high seed or anchors density employed. In [9], MSL/MSL* algorithm was implemented with an improved sampling procedure accompanied by high accuracy when nodes are static or mobiles (with reference to MCL) but at the expense of communication cost . MSL/MSL* is an optimized version of MCL. APIT proposed by [10] uses the point-in-triangulation technique, but high anchor density and long radio range of anchors are required. The multidimensional scaling(MDS) approach, [17], relies on central processing node that collates and perform computation of location assignments but has a problem of scalability. However distributed version of MDS has been proposed [16].

Cooperative localisation techniques, based on multidimensional scaling (MDS), concern distributed approaches for nodes' locations estimation either in range based or range free conditions. As reported in [13], with minimal number of anchors are required, such approaches can deliver node position accuracy $<20 \% r$, with $r$ the average radio signal radius. The main question concerns time complexity and the meaning of $\mathrm{r}$ regarding an error reported to the real node location.

In [13], the authors present a new approach for cooperative node localisation, using a neural networks projection method called CCA, for Curvilinear Component Analysis. The main idea of the CCA method concerns the projection- reduction between an input space of dimension $N \times N$ to an output space of dimension $N \times M$, with $M<<N$, where $\mathrm{N}$ is the number of nodes. However, the reported complexity is about $O\left(N^{2}\right)$. The proposed approach is closed to the approach proposed above, with the main difference concerning locality exploitation of the join information exchanged by nodes in the same vicinity leading to a complexity of $O(N)$.

\section{THE PROPOSED APPROACH}

At the network initialisation some control data are exchanged between the nodes and the relevant sinks (Access Points), depending on the application area. Some of such information are dedicated to network topology identification and nodes'localisation. Such data will not overload the network. As the control and data packets, using appropriate coding, will be used to send the localisation data.

The proposed approach proceed according to three phases:

\section{Phase 1 : Initialisation phase}

Considering a given node $i$, at the initialisation stage, such node starts discovering its neighbour and getting a path to a sink. The node sends to the sink:

1) the list of its neighbours $N g h\{i\}$;

2) the associated measured distances, if available, $\left[d_{i j}\right]$ with $j \in N g h\{i\}$.

Phase 2: Initial Positions estimation At the sink, the received data from all the nodes serves to compute the network topology (graph). Based on the node-to-node measured distances and the positions of a few nodes $(\leq 4)$ named anchors or GPS nodes, the sink compute an initial estimate of the nodes' locations.

The problem is formulated as minimum spanning tree problem and solved accordingly. The complexity is about $O(N \log (N)$ with $\mathrm{N}$ being the number of nodes in the network.

Then the sink sends to all nodes their initial positions, using some control packet as ACK packet for data packets. To improve the obtained initial solution, line search optimisation could be used at the sink, with a complexity of $O\left(N^{2}\right)$

Phase 3: Distributed approach for Positions estimation Based on the initial position and the neighbouring nodes' positions, each node compute its position based on the minimisation of the error between the measured distances, [14], and the computed ones. The complexity of the proposed approach is $O(N)$ for all the network. But as the approach is distributed and based on local information, the computation 
time is constant for each node. The node broadcasts its position to neighbouring nodes.

Phase 4: maintenance phase When the node looses the majority of its neighbouring nodes, it sends a new request to the sink to get a new estimate of its new position. The majority of neighbouring nodes is related to the minimum number of neighbouring nodes needed to estimate the node's position. If a given node, due to network topology modification (mobility, died nodes, new nodes, ...), lost the nodes in its vicinity, it must request a new global position estimation from the sink. The sink, based on the topology change, computes new estimates to the requesting nodes. In the same way, new nodes are seen as nodes that have changed their location.

\section{Modeling}

Given a WSN with the following characteristics

- $\mathrm{N}$ nodes with unknown positions, called sensors

- $\mathrm{K}, K<<N$ nodes with known position, called GPS nodes or anchors

- $M=N+K$ total number of nodes in the WSN

- $\mathrm{Br}$ total number of branches in WSN, representing nodes'connectivity

The number of nodes and branches may change during lifetime of the WSN.

The aim of the localisation approach is to match the computed distances based on nodes positions to the measured ones. The distance measurement is ensured using RSSI and nodes density in the vicinity of the actual node. If distance data are missing for some nodes, estimated values could be computed based on the network connectivity graph.

Let us consider two nodes, named sender(s) and receiver (r) nodes. A stage $\mathrm{k}$ if $\delta_{k}$ is the computed distance and $d_{k}$ the measured distance, in this case with have :

$$
\delta_{k}^{2}=\left(x_{s(k)}-x_{r(k)}\right)^{2}+\left(y_{s(k)}-y_{r(k)}\right)^{2} \approx d_{k}^{2}
$$

the approximation of the above equation leads to :

$$
2 \delta_{k} \partial \delta_{k}=\begin{aligned}
& 2\left[\left(x_{s(k)}-x_{r(k)}\right) d x_{s(k)}-\left(x_{s(k)}-x_{r(k)}\right) d x_{r(k)}\right. \\
& \left.+\quad\left(y_{s(k)}-y_{r(k)}\right) d y_{s(k)}-\left(y_{s(k)}-y_{r(k)}\right) d y_{r(k)}\right]
\end{aligned}
$$

thus,

$$
\begin{gathered}
\delta_{k} \partial \delta_{k}=+\begin{array}{c}
\left(x_{s(k)}-x_{r(k)}\right)\left(d x_{s(k)}-d x_{r(k)}\right) \\
+\left(y_{s(k)}-y_{r(k)}\right)\left(d y_{s(k)}-d y_{r(k)}\right)
\end{array} \\
\partial \delta_{k}=\frac{(\Delta x)_{k}}{\delta_{k}}\left(d x_{s(k)}-d x_{r(k)}\right)+\frac{(\Delta y)_{k}}{\delta_{k}}\left(d y_{s(k)}-d y_{r(k)}\right)
\end{gathered}
$$

In the global form, considering all the nodes, we have:

$$
d \delta=D_{x} C d X+D_{y} C d Y \approx d-\delta
$$

with

$$
D_{x}=\operatorname{diag}\left\{\frac{(\Delta x)_{k}}{\delta_{k}}\right\}
$$

$$
\begin{gathered}
D_{y}=\operatorname{diag}\left\{\frac{(\Delta y)_{k}}{\delta_{k}}\right\} \\
d X=\left[d X_{i}\right] \\
d Y=\left[d Y_{i}\right]
\end{gathered}
$$

and $\mathrm{C}$ is the incidence matrix given by:

$$
C=\left[C_{k i}\right]
$$

with

$$
C_{k i}=\left\{\begin{aligned}
1 & \text { if } i=s(k) \\
-1 & \text { if } i=r(k) \\
0 & \text { otherwise }
\end{aligned}\right.
$$

\section{NEIGHBOUR BASED LOCALISATION}

For any node (sensor) $i$ in the WSN,

- the set of Neighbouring nodes is $N g h\{i\}$

- its actual position is $x_{i}(k)$ given in 2-D plan

- the positions of the neighbouring nodes are given by : $x_{j}(k), j<N g h\{i\}$.

- the set of measured distances between node $i$ and the neighbouring nodes are given by : $d_{i j}, j \in N g h\{i\}$ and $i \in N d$ (set of $\mathrm{N}$ nodes)

The local schema for updating the nodes' positions is given by:

1) Step 1: Initialisation $k=0$

2) Step 2:Pre-computing

$$
\begin{aligned}
& \forall j \in N g h\{i\}: \\
& \delta_{i j}(k)=x_{i}(k)-x_{j}(k) \\
& \hat{d}_{i j}(k)=\sqrt{<\delta_{i j}, \delta_{i j}>} \\
& f_{i j}(k)=\frac{d_{i j}(k)-\hat{d}_{i j}(k)}{d_{i j}(k)} \\
& \alpha(k)=\beta(k) \times 1 / \sum_{j} f_{i j}(k) \mid
\end{aligned}
$$

with $\beta(k)$ a monotonic decreasing function $\Delta x y_{i}=\delta_{i} f_{i}$ with $\delta_{i}=\left[\delta_{i j}\right]$ and $f_{i}=\left[f_{i j}\right]$

3) Step 3: Updating

$$
x_{i}(k+1)=x_{i}(k)-\alpha(k) \times \Delta x y_{i}
$$

4) Step 4: propagation Each node propagate to its neighbour its new position

5) Step 5: Stopping criteria

If cost $<\epsilon$ than stop

elsewhere $k \leftarrow k+1$ goto to Step2

cost is related to the distance error as follows :

$$
\operatorname{cost}=\|d(k)-\hat{d}(k)\|
$$

The convergence is ensured if the error between the measured distance and the computed ones are within some admissible value. 


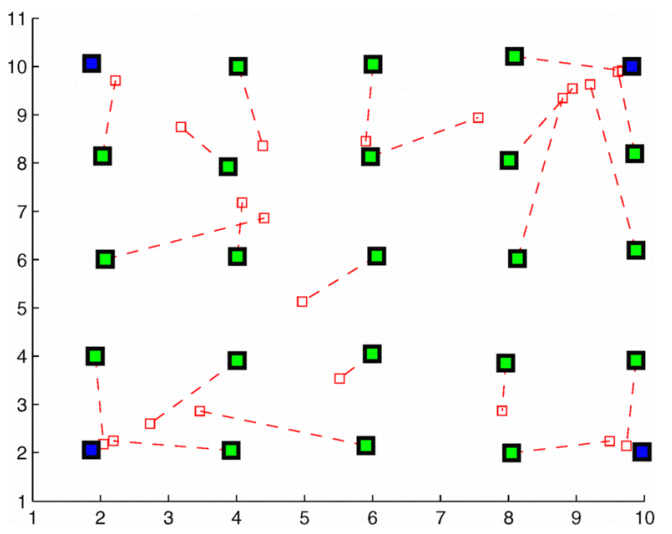

Fig. 2. Initial Positions estimation (Phase 2) with WSN of 25 Nodes

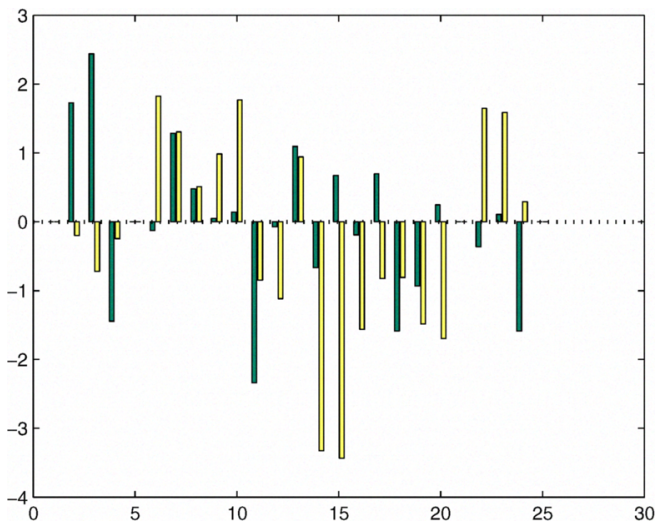

Fig. 3. Positions Errors (Phase 2)

\section{SimULATION RESULTS}

We consider a WSN with a 25 nodes, with 4 anchors. The following Figures $(2-5)$ show the obtained results for the initialisation phase using minimum spanning tree and the distributed local approach with the associated position errors. The blue square represent the anchors'positions, the green ones represent the real nodes' positions and the red ones the estimated nodes' positions.

The figure (6) shows the measured distance and the real distance, in order to show the robustness of the approach regarding errors in distance measurement.

The presented results show the effectiveness of the proposed approach regarding the accuracy.

Considering a network with 96 nodes and 4 GPS nodes,at the same position as for the first simulation scenario, i.e. the four nodes at the four corners of the area, the figure (7) shows the initial positions obtained by our spanning tree algorithm and figure 8 shows the results obtained after 5 iterations of the local algorithm. Based on available information at each stage, each iteration concerns position computation by each node and

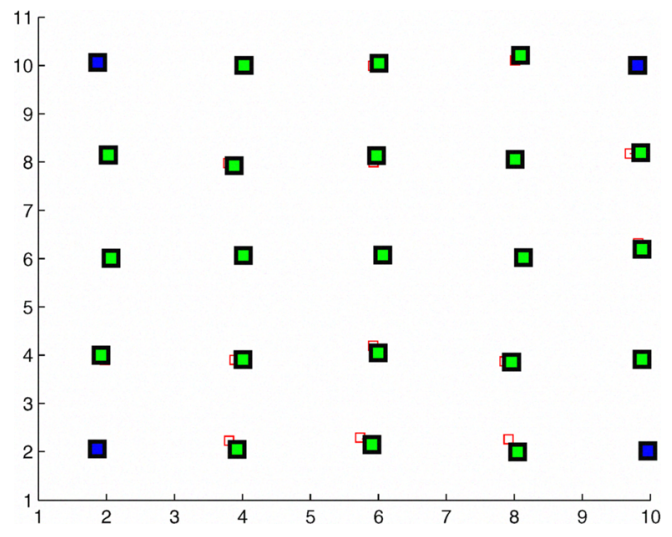

Fig. 4. Distributed Positions estimation (Phase 3) with WSN of 25 Nodes

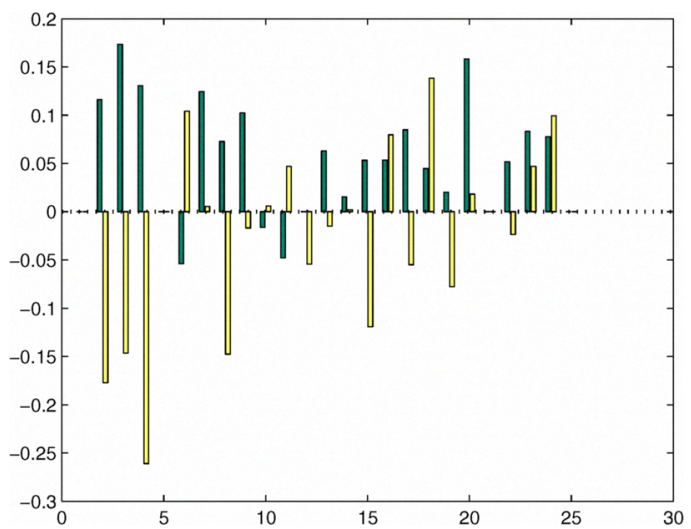

Fig. 5. Positions Errors (Phase 3) with WSN of 25 Nodes

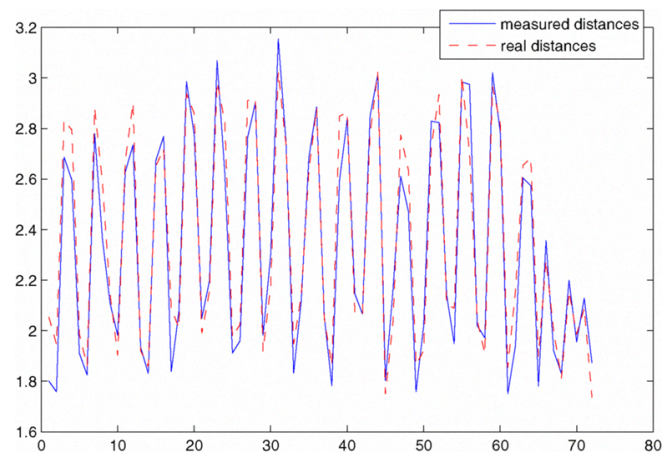

Fig. 6. Measured and Real distance

positions broadcasting to neighboring nodes (maximum of 5).

\section{CONCLUSION}

This paper focused on nodes' localisation in wireless sensor network. After discussing the main contributions in the literature we proposed a distributed approach that exhibits accuracy 


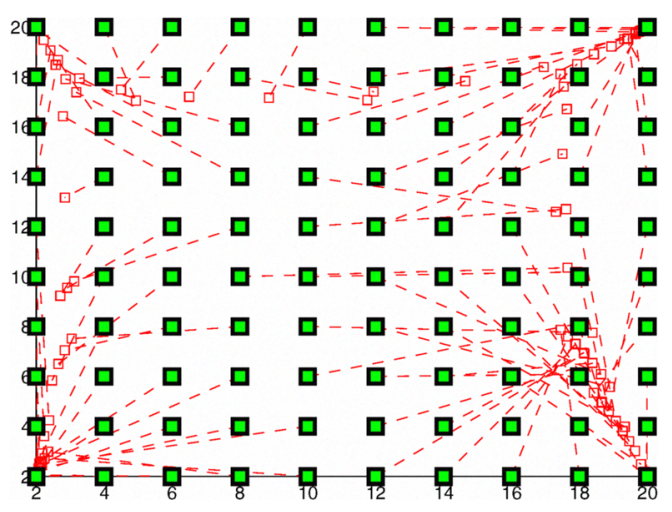

Fig. 7. Initial Positions estimation (Phase 2) with WSN of 100 Nodes

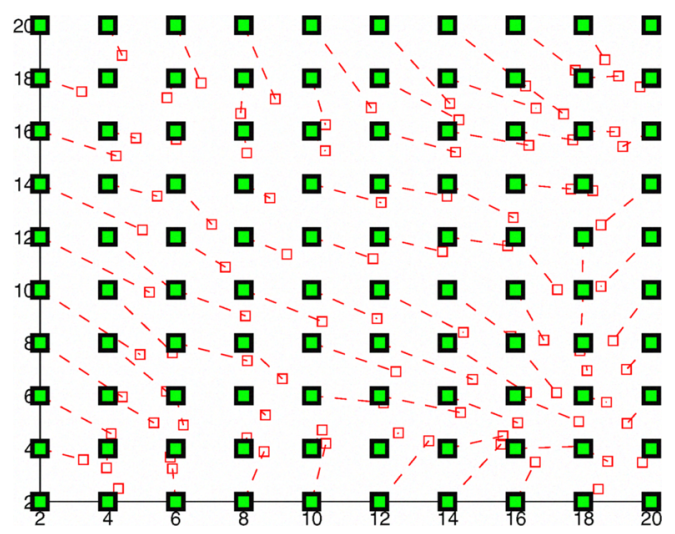

Fig. 8. Distributed Positions estimation (Phase 3) with WSN of 100 Nodes

and scalability with computation efficiency. On the scalability issue, the proposed approach is local, so the complexity is bounded by the number of nodes used for the position estimation. However the initial phase which is performed at the sink has a complexity of $\mathrm{O}(\log (\mathrm{N}))$.

The work is ongoing mainly on theoretical part to show that position estimation in dynamic environment is equivalent to a differential game and on algorithm part to address convergence and complexity analysis regarding several scenarios.

\section{REFERENCES}

[1] Michael Carter, Holly Jin, Michael Saunders and Yinyu Ye, SPASELOC: An Adaptive Subproblem Algorithm for Scalable Wireless Sensor Network Localization, SIAM Journal on Optimization, Vol. 17, No 4, pp. 1102-1128, 2006.

[2] L. Doherty, L. El Ghaoui, and K. Pister, Convex position estimation in wireless sensor networks, in Proceedings of the IEEE Infocom 2001, Anchorage, AK, pp. 1655-1663, 2001.

[3] P. Biswas and Y. Ye, Semidefinite programming for ad hoc wireless sensor network localization, in Proceedings of the Third International Symposium on Information Processing in Sensor Networks, Berkeley, CA, 2004.

[4] P. Tseng, SOCP relaxation for non convex optimization, ICCOPT 1, Rensselaer Polytechnic Institute, Troy, NY, 2004.
[5] P. Biswas and Y. Ye, A distributed method for solving semidefinite programs arising from ad hoc wireless sensor network localization, in Multiscale Optimization Methods and Applications, Non convex Optim. Appl. 82, Springer, New York, 2006.

[6] Cong Tran-Xuan, Eunchan Kim and Insoo Koo, An Efficient RSS-Based Localization Scheme with Calibration in Wireless Sensor Networks, IEICE Transactions on Communications, E91-B(12):4013-4016, 2008.

[7] A. Boukerche, H.A.B.Oliveira, E.F.Nakamura and A.A.F. Loureiro, $A$ Voronoi Approach for Scalable and Robust DV-Hop Localization System for Sensor Networks, Computer Communications and Networks, 2007. ICCCN 2007. Proceedings of 16th International Conference on Volume , Issue , 13-16 Aug. 2007 Page(s):497 - 502.

[8] L. Hu and D. Evans, Localization for mobile sensor networks, in the proceedings of the MobiCom'2004, pages 1-13, Sep-Oct 2004.

[9] M. Rudafshani and S. Datta, Localization in wireless sensor networks, in the proceedings of the IPSN'07, pages 51-60, 2007

[10] Tian He, Chengdu Huang, Brian M. Blum, John A. Stankovic, and Tarek Abdelzaher, Range-free localization schemes for large scale sensor networks, in the proceedings of the Mobicom 2003, pages 81-95, Se 2003.

[11] D. Niculescu and B. Nath, Dv based positioning in ad hoc networks, In Journal of Telecommunication Systems, 2003

[12] Jeongkeun Lee, Kideok Cho, Seungjae Lee, Taekyoung Kwon, Yanghee Choi, Distributed and energy-efficient target localizayion and tracking in wireless sensor networks, Computer Communication, pp 2494-2505, 2006.

[13] Li Li and Thomas Kunz, Cooperative Node Localization Using Nonlinear Data Projection, ACM Transaction on Sensor Networks, Vol. 5, No. 1, Feb. 2009

[14] Urs Bischoff, Martin Strohbach, Mike Hazas and Gerd Kortuem, Constraint-Based Distance Edtimation in Ad-Hoc Wireless Sensor Networks, EWSN 2006, LNCS 3868, pp. 54-68, 2006.

[15] Radu Stoleru, Jihn A. Stankovic, and Sang Son , Robust node localization for wireless Sensor Networks, 4th Workshop on Embedded Networked Sensor (EMNets '07), ACM, June , pp. 48-52, 2007.

[16] Y. Shang, W. Ruml, Y Zhang, M.P.J. Fromherz, Location for more Connectivity, 4th ACM International Symposium on Mobile Ad hoc Network and Computing (MobiHoc 2005), 2005.

[17] X. Ji and H. Zha, Sensor Positioning in Wireless ad hoc Sensor Networks Using Multidimensional Scaling, 23rd Annual Joint Congress of IEEE Computer and Communication Society (INFOCOM 2004), 2004. 